\title{
Genetic Polymorphism in Peripartum Cardiomyopathy
}

\author{
Ivana Purnama DEWI $I^{1,2,3^{*}}$, Johanes NUGROHO ${ }^{1,3}$
}

Surabaya, Indonesia

\begin{abstract}
Peripartum cardiomyopathy is a rare type of cardiomyopathy. Peripartum cardiomyopathy is a potentially life-threatening pregnancy-associated disease that typically arises in the peripartum period and is marked with left ventricular dysfunction and heart failure. The cause of peripartum cardiomyopathy remains unclear, but several mechanisms have been proposed which indices a potentially multi-factorial etiologies. Early case reports identified overlap between familial dilated cardiomyopathy and peripartum cardiomyopathy, although the degree of overlap is largely unknown. Evidence supporting a contribution from gene mutations in peripartum cardiomyopathy includes genome-wide association studies, familial occurrence, variable prevalence among different regions and ethnicities, and more recent investigations of panels of genes for mutations among women with peripartum cardiomyopathy. Although the true incidence of genetic cardiomyopathy is not yet known among women with peripartum cardiomyopathy, there is substantial evidence demonstrating that genetic contribution to their condition.
\end{abstract}

Keywords: Familial dilated cardiomyopathy, Genetic, Peripartum cardiomyopathy, Pregnancy

Gynecol Obstet Reprod Med 2021;27(3):297-301

\section{Introduction}

Peripartum cardiomyopathy (PPCM) was first described in the mid-19th century as a post-partal disease that links acute heart failure and the puerperium (1). However, it was only in 1937, when Gouley et al. wrote the clinical characteristics and pathological description of seven pregnant patients, that PPCM was first considered as clinical with different pathophysiology. These seven pregnant patients were diagnosed as having dilated cardiomyopathy (DCM) without ischemic disease by the end of their pregnancy and remained the same until their post-delivery. Four of these seven patients died soon after the diagnosis and the autopsy indicated heart dilatation with areas of necrosis and fibrosis (2).

Faculty of Medicine, Universitas Airlangga, Surabaya, Indonesia

${ }^{2}$ Faculty of Medicine, Duta Wacana Christian University, Yogyakarta, Indonesia

${ }^{3}$ Department of Cardiology and Vascular Medicine, Dr. Soetomo General Hospital, Surabaya, Indonesia

Address of Correspondence: Ivana Purnama Dewi

Department of Cardiology and Vascular

Medicine, Faculty of Medicine, Universitas

Airlangga - Dr. Soetomo General Hospital,

Surabaya

drivanapd@staff.ukdw.ac.id

Submitted for Publication: 18.02.2020 Revised for Publication: 22.06.2020 Accepted for Publication: 14.03.2021 Online Published: 31.03.2021

ORCID IDs of the authors: IPD: 0000-0002-1602-3384

$J N: 0000-0002-3501-1872$

\begin{tabular}{|c|c|}
\hline \multirow{3}{*}{ 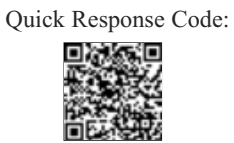 } & Access this article online \\
\hline & $\begin{array}{l}\text { Website: www.gorm.com.tr } \\
\text { e- mail: info@gorm.com.tr }\end{array}$ \\
\hline & DOI:10.201613/GORM.2021.1072 \\
\hline
\end{tabular}

How to cite this article: Dewi IP. and Nugroho J. Genetic Polymorphism in Peripartum Cardiomyopathy. Gynecol Obstet Reprod Med. 2021;27(3):297301
Peripartum cardiomyopathy is the most common cardiomyopathy in pregnancy. PPCM is defined as idiopathic cardiomyopathy, associated with pregnancy, with characteristics of secondary heart failure caused by left ventricular (LV) systolic dysfunction near the end of pregnancy or in the months after delivery (3). PPCM is a suspected diagnosis when there are no other causes of heart failure, where left ventricular dilatation may not occur, but the ejection fraction will always decrease below $45 \%$. There are 4 criteria to explain the definition of PPCM, i.e.

1. Heart failure happened in the last month of pregnancy or at five months postpartum

2. Other causes of heart failure could not be identified

3. No history of heart disease is obtained before pregnancy

4. Left ventricular systolic dysfunction (LVSD) confirmed by echocardiography with the criteria for the left ventricular ejection fraction was $<45 \%$ or the fractional shortening was $<30 \%$ or both of those criteria were met, with or without the end-diastolic ventricular dimensions were $>2.7 \mathrm{~cm} / \mathrm{m} 2$ at body surface area (4).

Another term is early pregnancy cardiomyopathy (PACM), which is explained as cardiomyopathy that occurs before the last month of pregnancy. A study that assessed the clinical characteristics of 23 women who suffered from the PACM (the earliest diagnosis was reported at week 17th of pregnancy) compared to 100 women who suffered from PPCM revealed no clinical difference in both cardiomyopathies (PACM and PPCM). Phenotypically, it is difficult to distinguish PPCM from PACM due to DCM where systolic function dysfunction and LV dilatation occur (5). 
Peripartum cardiomyopathy is a rare disease but its incidence is reported to increase continually. The PPCM incidence varies considerably in various countries. In Asian countries the PPCM incidences were reported in the following countries; in Singapore with a ratio of 0.89:1000, in Japan with a ratio of $1: 1000$, in Pakistan with a ratio of $1: 837$, at Indian tertiary hospital with a ratio of 1:1374, and in Malaysia with a ratio $34: 100,000$ of live births. At present, there is no national data available on the PPCM incidence in Indonesia. Epidemiological data indicates the incidence of PPCM is much higher in Haiti (1:300), Nigeria (1\% of live births), South Africa (1:1000), and Afro-Americans in the United States (US), 1:1000-4000. Based on these epidemiological data, it is possible to conclude that African is a risk factor for PPCM. Although environmental factors can also influence genetics to have an important role because PPCM incidence remains higher for Africans who move to the US. A study by Gentry et al reported that the incidence of PPCM was 16 times higher in Afro-Americans compared to non-Africans who living in Georgia and Tennessee (6).

Until now, there are many hypotheses regarding the etiology of PPCM but none have been able to be used as the main explanation for all cases of PPCM. PPCM is known to have pathogenesis which involves many factors. These factors include; maternal autoimmune responses, inflammation, increased oxidative stress, micronutrient deficiency, imbalance of cardiac proptosis factors, antiangiogenic factors, viral infections, and genetic factors (7). Because of the PPCM complexity, genetic roles can be the etiology of PPCM ranging from the spectrum of relatively common variants with smaller effects to rare variants with large effects. The purpose of this literature review is to conclude various evidence on the role of genetics in the pathogenesis of PPCM, as well as the related pathways involved.

\section{Peripartum cardiomyopathy as a subgroup of familial di- lated cardiomyopathy}

Several cohort studies revealed a positive family history of DCM in $10 \%$ of PPCM patients. In some case reports, it was found that PPCM can be a familial dilated cardiomyopathy (FDC). It was reported that women with PPCM have a mother or sister who is diagnosed with PPCM also, some have reported an association between first-degree relative sexes of women. Women who have the DCM gene can suffer from PPCM after pregnancy due to hemodynamic stress.

There are two studies looking at patterns of genetic inheritance in PPCM. The first study by van Spaendonck-Zwarts et al. reviewed the genetic component of PPCM and tested the hypothesis that PPCM is part of the FDC that emerged in the peripartum period (8). This study employs two methodologies: 90 families with a history of idiopathic DCM (IDCM) and 10 PPCM patients. It indicated that FCM was diagnosed when there were two or more family members who suffered from DCM, or there was a relative first-degree presence of
DCM patients who died suddenly before 35 years. Based on the evaluation of 90 families with IDCM, it was found that five families $(6 \%)$ had at least one individual with PPCM. Based on the screening evaluation in the family of ten PPCM patients, it was found three patients with relative first-degree who were not diagnosed with DCM. The DNA analysis in five IDCM families and three PPCM patients did not reveal DCMrelated gene mutations such as LMNA, TNNT2, MYH7. One of the five IDCM families had extensive DNA testing and found c.149A $>$ G, p.Gln50Arg mutations in cardiac troponin C (TNNC1) (8).

The second study was performed by Morales et al., by using the FDC Research Project cohort database, with its study subject taken from family subjects with FDC and FDC. A total of 4110 women from 520 family trees were reviewed and 45 cases of PPCM / PACM were obtained from two sibling relations, two cousins' relation, and two nieces-nephews' relations, while the rest 42 cases having no family relations. Among them, 23 cases (55\%) were the familial case and 12 cases $(29 \%)$ were sporadic, while seven others did not get any sufficient data. The 19 subjects met PPCM criteria while eight others met PACM criteria. Genetic data from sequencing studies performed in the 19 cases indicated 13 cases were familial and the rest 6 cases were sporadic. Gene sequencing analysis found six $(32 \%)$ cases which consist of five PPCM and one PACM, carrying non-identical mutations, from different genes. In four familial cases, the mutations were found in MYH7, PSEN2, MYBPC3, and SCN5A. In two sporadic cases, the mutations were found in TNNT2 and MYH6 (5). These two studies were the beginning of research on PPCM. The limitations of the two studies are as follow:

1. The limited sample for genetic analysis

2. The absence of genetic screening and extensive clinical evaluation in families of the patients who have recovered their LV function

However, the clinical implications of these two studies were the recommendations for employing pre-symptomatic screening for IDCM for all first-degree relatives of the patients who suffered from PPCM and did not recover their LV size and function. Cardiologic screening during pregnancy until the puerperium period can be considered to be performed for healthy women who are IDCM patients' first-degree relatives (4).

\section{Peripartum cardiomyopathy related to titin mutation}

In recent years, TTN has received much attention for its role in DCM and several skeletal muscle diseases, such as limb griddle muscular dystrophy type 2J (LGMD2J), tibialis muscular dystrophy (TMD), and hereditary myopathy with early respiratory disorders (9). TTN encodes a human's largest protein, titin, which is the third-largest protein in striated muscle.

Titin has an important role in the sarcomere's organization 
and assembly, as the structural elements ranging from Z-disks to M-lines (Figure 1) (10). Because TTN is an important factor for the structure, development, mechanical function, and heart and skeletal muscle regulation, it is important to understand TTN as a genetic cause for various diseases as mentioned above. Its large size (363 exons) correlates with the high likelihood of mutation in this gene.

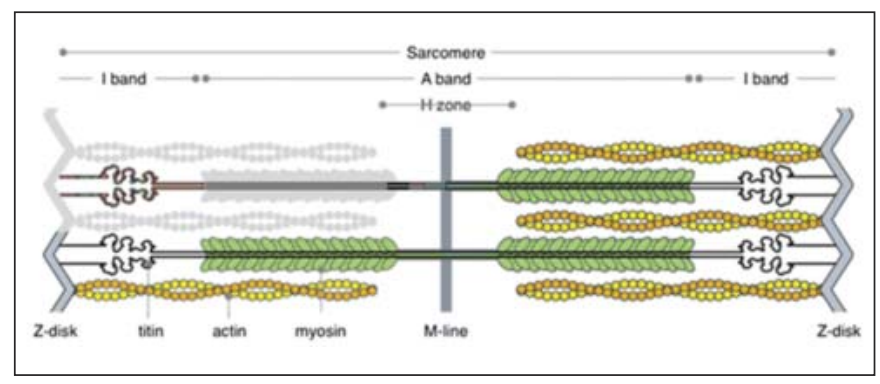

Figure 1: A Sarcomere Structure (11)

An early TTN study on 312 non-ischemic DCM indicated a mutation cut in the TTN at 67/312 (21\%) especially at the Aband. At 7/249 (3\%) control reported without cardiomyopathy and $3 / 231(1 \%)$ people reported to have hypertrophic cardiomyopathy (HCM) from cutting in this gene. Three people with HCM and TTN mutations also had an MYH7 or MYBPC3 mutation. In contrast to DCM, the majority of TTN cuts (without cardiomyopathy or with HCM) occur in the Nterminal section (12).

Genetic studies were then conducted to determine the relationship between PPCM and FDC. A study in the Netherlands analyzed 48 genes in 18 families with PPCM and DCM. They identified four pathogenic cuts in 4 of 18 families (22\%), three in TTN and one in BAG3, as well as six other variants of unknown significance (VUS) that might be pathogenic (33\%). Four of the VUSs were cuts on TTN located in the A-band, thus a high prevalence of TTN cuts in PPCM 7/18 (39\%) was obtained (13).

Another study was conducted by analyzing 43 genes in 172 women with PPCM. The genetic evaluation employed DNA sequencing, which focused more on mutations due to the variant cuts rather than from the missense alleles. In this study, the cutting mutations at eight genes were identified from the 26/172 (15\%). The prevalence of TTN cuts was then compared to the ones in DCM patients and the control population. The results were consistent with previous studies, where the mutation of TTN cuts in PPCM was 10\% (17/172), which was higher than the control group. The heterozygous cuts in two genes on the $\mathrm{X}$ chromosome (DMD and LAMP2), as well as cuts in DSP, MYH6, SYNM, TPM1, and VCL were identified also. Among these genes, MYH6 and SYNM have a mutation cut of more than $0.5 \%$ (14). Although the prevalence of cutting mutations in genes could indicate the possibility of overestimating the analysis of genetic DCM events among women with PPCM, it is important to recognize that many of the analyzed genes usually had pathogenic missense mutations in addition to the cutting mutations that caused DCM.

\section{Peripartum cardiomyopathy in women with Carrier X- linked Cardiomyopathy}

The 2001 case report reported that a 25-year-old young woman, who suffered from PPCM in the 36th week of pregnancy until after delivery, needed a left ventricular device (LVAD) due to severe LV systolic dysfunction and eventually underwent a cardiac transplant (15). She was identified to have a medical history of Duchenne Muscular Dystrophy (DMD) based on her medical record. During the LVAD insertion, a widening of the entire cardiac chamber was consistent with acute exacerbation of chronic cardiomyopathy. However, it was not clear whether this patient had undiagnosed cardiomyopathy due to her carrier condition for DMD or whether she had PPCM which was not related to her carrier status, but the patient met the diagnostic criteria for PPCM because she had no symptoms of heart failure before the last month of her pregnancy.

A 1996 cohort study observed the development of cardiomyopathy in women with DMD and Becker Muscular Dystrophies (BMD). The results showed that there was preclinical or clinical myocardial involvement in $84.3 \%$ of the total cohort (166/197 cases), without significant differences in percentages and phenotypes between DMD and BMD carriers (16). A case report reported a 40-year-old woman diagnosed and treated for PPCM was found to be a carrier of the DMD gene mutation after her son was diagnosed with DMD at the age of 4 years (17).

Ware et al., performed a study on two individuals who had mutational mutations in DMD and LAMP2, and it identified a relationship between these gene mutations and PPCM due to the imbalance of $\mathrm{X}$ chromosome inactivation (14). Two other case reports identified individuals with PPCM who had a DMD or LAMP2 mutation, hereinafter referred to as Danon disease. The women as Danon disease carriers were reported having cardiomyopathy and heart failure with an average age of heart transplantation at $32.3 \pm 14.5$ years and a mortality rate of 40.2 \pm 12.6 years (18). The incidence of PPCM among women with heterozygous LAMP2 mutations was unknown, it was due to the small incidence of Danon disease. However, the presence of LAMP2 mutations had a relationship with the progression of the development of heart disease in pregnant women.

\section{Other theories on the pathophysiology of Peripartum cardiomyopathy}

One of the current PPCM pathogenesis theories is the "two-hit hypothesis" which believes the vascular effect on cardiomyopathy is caused by anti-vascular or hormonal effects in the late pregnancy or early postpartum period. Other studies had identified the severity of PPCM characterized by cardiac angiogenic imbalance. 
Signal Transducer and Activator of Transcription-3 (STAT3) is a transcriptional factor that is widely studied. STAT3 is an acute phase response factor that has a vital role in regulating many genes involved in cell survival, hypertrophic growth modulation, angiogenesis, development and regeneration, energetics and metabolic reserves, antioxidant pathways, the composition of the extracellular matrix, and inflammatory processes (21).

One of STAT3's cardioprotective functions is to reduce oxidative stress by increasing the enzyme Reactive Oxygen Species (ROS), Manganese Superoxide Dismutase (MnSOD). Studies on mice that did not have the STAT3 gene in their cardiomyocytes indicated that the mice might suffer from cardiomyopathy (21). The loss of STAT3 suppresses the expression of manganese superoxide dismutase (MnSOD), the substance that protects against ROS. The loss of MnSOD expression causes an increase in the cathepsin D peptidase enzyme, an enzyme that breaks down prolactin, anti-angiogenic, and proapoptotic fragment $16-\mathrm{kDa}$, called vasoinhibin, which can cause myocardial damage and ventricular dysfunction through cellular apoptosis and capillary dropping (21,22). Bromocriptine, a dopamine receptor antagonist, shows potential therapeutic results in increasing LV function in the PPCM population (Figure 2).

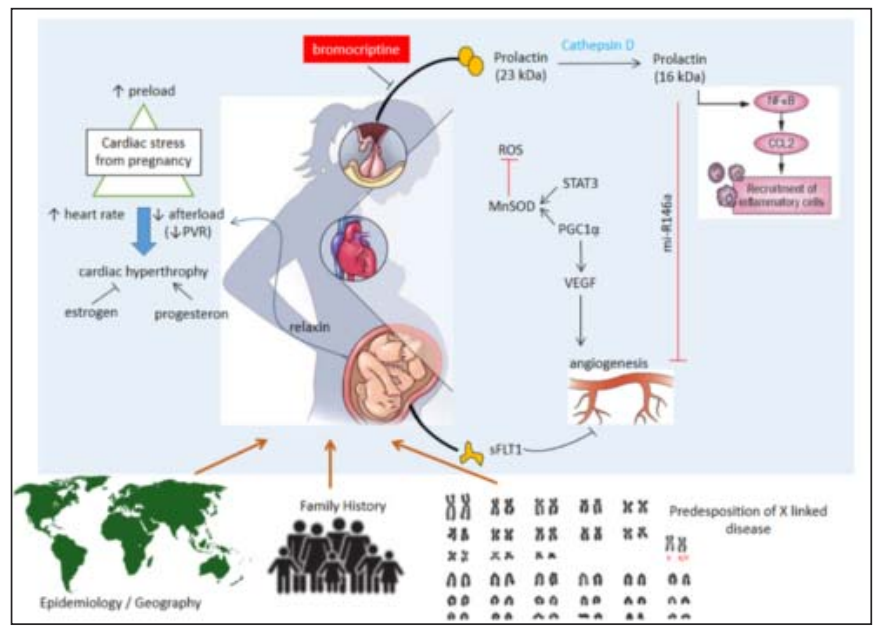

Figure 2: The etiological factors and pathogenesis of Peripartum cardiomyopathy $(19,20)$

Proliferator activated gamma coactivator-1alpha (PGC1alpha), a transcription coactivator that is very important in mitochondrial biogenesis and expression and secretion of proangiogenic factors such as vascular endothelial growth factor (VEGF). Just like STAT3, PGC-1alpha increases the ROS enzyme. Towards the end of pregnancy, the placenta systemically secretes soluble FMS-like tyrosine kinase receptor1 (sFLT-1). High sFLT-1 levels can cause cardiomyopathy (23).

\section{Conclusion}

Several studies support the substantial role of genetics for PPCM. From a variety of case reports to larger multicenter in- vestigations, they indicated that at least $10-15 \%$ of women with PPCM had gene mutations. Research on murine identified a relationship between the role of genes with metabolism and free radical stress. Future studies are expected to be able to look for the prevalence of pathogenic mutations that contribute to PPCM and also can help to provide prognostic information for women suffered from PPCM

Acknowledgments: Not applicable.

Funding: The authors received no financial support for the review, authorship, and/or publication of this article.

Author contribution: JN: Conceived the idea and designed the report. IPD: Was a major contributor in writing the manuscript. All authors read and approved the final manuscript. Conflict of interest: The authors declare that there is no conflict of interest.

\section{References}

1. Elkayam U, Akhter MW, Singh H, Khan S, Bitar F, Hameed A, et al. Pregnancy-Associated Cardiomyopathy. Circulation. 2005;111(16):2050-5. Doi: 10.1161/01.CIR. $0000162478.36652 .7 \mathrm{E}$.

2. Gouley BA, McMillan TM, Bellet S. Idiopathic myocardial degeneration associated with pregnancy and especially the puerperium. Am J Med Sci. 1937;19:185-99.

3. Regitz-Zagrosek V, Roos-Hesselink JW, Bauersachs J, Blomström-Lundqvist C, Cífková R, De Bonis $\mathrm{M}$, et al. 2018 ESC Guidelines for the management of cardiovascular diseases during pregnancy. Eur Heart J. 2018;39(34): 3165-241. Doi:10.1093/eurheartj/ehy340.

4. Demakis JG, Rahimtoola SH, Sutton GC, Meadows WR, Szanto PB, Tobin JR, et al. Natural course of peripartum cardiomyopathy. Circulation. 1971;44(6):1053-61. Doi: 10.1161/01.cir.44.6.1053.

5. Morales A, Painter T, Li R, Siegfried JD, Li D, Norton N, et al. Rare variant mutations in pregnancy-associated or peripartum cardiomyopathy. Circulation. 2010;121(20): 2176-82. Doi:10.1161/circulationaha.109.931220.

6. Gentry MB, Dias JK, Luis A, Patel R, Thornton J, Reed GL. African-American women have a higher risk for developing peripartum cardiomyopathy. J Am Coll Cardiol. 2010;55(7):654-9. Doi:10.1016/j.jacc.2009. 09.043.

7. Ntusi NB, Mayosi BM. Aetiology and risk factors of peripartum cardiomyopathy: A systematic review. Int J Cardiol. 2009;131(2):168-79. Doi:10.1016/j.ijcard.2008. 06.054 .

8. van Spaendonck-Zwarts KY, van Tintelen JP, van Veldhuisen DJ, van der Werf R, Jongbloed JDH, Paulus WJ, et al. Peripartum cardiomyopathy as a part of familial dilated cardiomyopathy. Circulation. 2010;121(20):216975. Doi:10.1161/circulationaha.109.929646.

9. Krul SP, van der Smagt JJ, van den Berg MP, Sollie KM, Pieper PG, van Spaendonck-Zwarts KY. Systematic re- 
view of pregnancy in women with inherited cardiomyopathies. Eur J Heart Fail. 2011;13(6):584-94. Doi:10. 1093/eurjhf/hfr040.

10. Gerull B. The rapidly evolving role of Titin in cardiac physiology and cardiomyopathy. Can J Cardiol. 2015; 31(11):1351-9. Doi: 10.1016/j.cjca.2015.08.016.

11. Tabish AM, Azzimato V, Alexiadis A, Buyandelger B, Knöll R. Genetic epidemiology of titin-truncating variants in the etiology of dilated cardiomyopathy. Biophys Rev. 2017;9(3):207-23. Doi:10.1007/s12551-017-0265-7.

12. Herman DS, Lam L, Taylor MRG, Wang L, Teekakirikul $\mathrm{P}$, Christodoulou D, et al. Truncations of Titin causing dilated cardiomyopathy. N Engl J Med. 2012;366(7):61928. Doi:10.1056/NEJMoa1110186.

13. van Spaendonck-Zwarts KY, Posafalvi A, van den Berg MP, Hilfiker-Kleiner D, Bollen IA, Sliwa K, et al. Titin gene mutations are common in families with both peripartum cardiomyopathy and dilated cardiomyopathy. Eur Heart J. 2014;35(32):2165-73. Doi: 10.1093/eurheartj/ ehu050.

14. Ware JS, Li J, Mazaika E, Yasso CM, DeSouza T, Cappola TP, et al. Shared genetic predisposition in peripartum and dilated cardiomyopathies. N Eng J Med. 2016;374(3):233-41. Doi:10.1056/NEJMoa1505517.

15. Davies JE, Winokur TS, Aaron MF, Benza RL, Foley BA, Holman WL. Cardiomyopathy in a carrier of Duchenne's muscular dystrophy. J Heart Lung Transplant. 2001;20(7):781-4. Doi:10.1016/s1053-2498(00)00240-0.
16. Politano L, Nigro V, Nigro G, Petretta VR, Passamano L, Papparella $\mathrm{S}$, et al. Development of cardiomyopathy in female carriers of Duchenne and Becker muscular dystrophies. JAMA. 1996;275:1335-8. Doi:10.1001/jama. 1996. 03530410049032.

17. Cheng VE, Prior DL. Peripartum cardiomyopathy in a previously asymptomatic carrier of Duchenne muscular dystrophy. Heart Lung Circ. 2013;22(8):677-81. Doi:10. 1016/j.hlc.2012.11.015.

18. Boucek D, Jirikowic J, Taylor M. Natural history of Danon disease. Genet Med. 2011;13(6):563-8. Doi:10. 1097/GIM.0b013e31820ad795.

19. Arany Z, Elkayam U. Peripartum Cardiomyopathy. Circulation. 2016;133(14):1397-409. Doi: 10.1161/c1rculationaha.115.020491.

20. Lee YZJ, Judge DP. The Role of Genetics in Peripartum Cardiomyopathy. J Cardiovasc Transl Res. 2017;10(5-6): 437-45. Doi:10.1007/s12265-017-9764-y.

21. Hilfiker-Kleiner D, Kaminski K, Podewski E, Bonda T, Schaefer A, Sliwa K, et al. A cathepsin D-cleaved $16 \mathrm{kDa}$ form of prolactin mediates postpartum cardiomyopathy. Cell. 2007;128(3):589-600. Doi:10.1016/j.cell.2006.12.0 36.

22. Honigberg MC, Givertz MM. Peripartum cardiomyopathy. BMJ. 2019;364:k5287. Doi:10.1136/bmj.k5287.

23. Yang Y, Rodriguez JE, Kitsis RN. A microRNA links prolactin to peripartum cardiomyopathy. J Clin Invest. 2013;123(5):1925-7. Doi:10.1172/JCI69286. 